\title{
Severe Reversible Neurological Complications following Amantadine Treatment in Three Elderly Patients with Renal Insufficiency
}

\author{
Miho Nakata Shoichi Ito Wakako Shirai Takamichi Hattori \\ Department of Neurology, Graduate School of Medicine, Chiba University, Chiba, Japan
}

Dear Sir,

Amantadine hydrochloride is widely used to treat Parkinson's disease. In Japan, amantadine is also commonly used to treat post-stroke depressive states and influenza A virus infection. There are a few reports describing amantadine toxicity in aged patients with renal dysfunction, but the neurological features of amantadine toxicity are not well recognized and thus might be misdiagnosed. Here, we present 3 patients with amantadine toxicity.

Case Reports (table 1)

Patient 1: A 70-year-old woman with a 9-year history of Parkinson's disease received daily oral treatments of $150 \mathrm{mg}$ amantadine hydrochloride and $200 \mathrm{mg}$ levodopa. She developed progressive renal dysfunction due to chronic glomerulonephritis in 1998, and hemodialysis was introduced in January 2002. Around this period, she developed poor communication, visual hallucinations, gait disturbance, and general myoclonic jerks in her limbs and trunk, without daily fluctuations. She became unable to stand up in March 2002, and she was then admitted to our hospital.

On admission, she was febrile and somnolent. She complained of restlessness, disturbed concentration, memory decline, and concrete visual hallucinations. Myoclonic jerks were present in her limbs and trunk at rest, and were exaggerated during motion. There was no apparent motor paresis or cerebellar ataxia. She was always grasping objects, suggesting marked grasp reflexes in both hands. Parkinsonism symptoms, such as rigidity, were mild, but she could not stand or walk.

Brain computed tomography showed no apparent ischemic changes, and electroencephalography showed no obvious paroxysmal discharges or triphasic waves. Her plasma amantadine hydrochloride level was high $(4,900 \mathrm{ng} / \mathrm{ml})$, and she was eventually diagnosed with amantadine toxicity. After cessation of oral amantadine hydrochloride, all of her symptoms and signs gradually improved; about 3 weeks later she was able to walk without aid.

Patient 2: A 74-year-old woman had a right cerebellar hemorrhage in 1991. She subsequently developed apathy, depression, and gait disturbance, and was treated with $200 \mathrm{mg}$ amantadine hydrochloride and $200 \mathrm{mg}$ droxidopa per day. In March 2005, she began to have visual disturbances, e.g., 'the floor looks crooked'. In April 2005, she developed fever, vomiting, and myoclonic jerks in her limbs, and became unable to stand independently.

On May 18, 2005, she was referred to our hospital. She did not speak voluntarily and was bound to her wheelchair. Neurological examinations revealed myoclonic jerks in limbs and marked grasp reflexes in both hands. There was no apparent motor paresis or cerebellar ataxia. Assessment of muscular tone was difficult because of declined auditory comprehension. Electroencephalography showed no obvious paroxysmal discharges or triphasic waves.
Blood examinations revealed mild renal dysfunction and/or dehydration (blood urea nitrogen: $42.5 \mathrm{mg} / \mathrm{dl}$, creatinine: $1.3 \mathrm{mg} / \mathrm{dl})$. Although her serum amantadine hydrochloride level was not evaluated, these clinical and laboratory features suggested a diagnosis of amantadine toxicity, and oral administration of amantadine hydrochloride was stopped. Her symptoms and signs gradually improved, and after about 3 weeks, she was able to walk without aid.

Patient 3: A 73-year-old woman with a 3-year history of Parkinson's disease had suffered from diabetic nephropathy since 1999, and was admitted to a hospital for dialysis treatment in January 2002. On February 17, 2005, she began treatment with oral $300 \mathrm{mg}$ amantadine hydrochloride per day for parkinsonism. She gradually developed somnolence and gait disturbance, and was bedridden about 1 week after onset. On March 6, 2005, amantadine toxicity was strongly suspected and her amantadine hydrochloride regimen was discontinued. General myoclonic jerks appeared. On March 12, 2005, she had an acute onset of high-grade fever, coma, and status epilepticus. A malignant syndrome was suspected, and treatment with dantrolene sodium was introduced. The treatment alleviated her fever within several days, but somnolence and dementia remained and she became bedridden. She was referred to our hospital and was admitted on August 11, 2005.

\section{KARGER}

Fax +4161306 1234 E-Mail karger@karger.ch www.karger.com
(C) 2006 S. Karger AG, Base 0014-3022/06/0561-0059\$23.50/0

Accessible online at: www.karger.com/ene
Shoichi Ito, MD, Department of Neurology

Graduate School of Medicine, Chiba University

1-8-1 Inohana, Chuo-ku, Chiba 260-8670 (Japan)

Tel +81 43226 2126, Fax +81 432262160

E-Mail sito@faculty.chiba-u.jp 
Table 1. Clinical features of 3 patients with amantadine toxicity

\begin{tabular}{|c|c|c|c|}
\hline & Patient 1 & Patient 2 & Patient 3 \\
\hline Age, years & 70 & 74 & 73 \\
\hline Gender & Female & Female & Female \\
\hline Neurological background & $\mathrm{PD}$ & $\begin{array}{l}\text { Post-stroke } \\
\text { depression }\end{array}$ & $\mathrm{PD}$ \\
\hline Renal dysfunction & $\begin{array}{l}\text { Severe (on } \\
\text { hemodialysis } \\
\text { due to CGN) }\end{array}$ & Mild & $\begin{array}{l}\text { Severe (on } \\
\text { hemodialysis } \\
\text { due to DN) }\end{array}$ \\
\hline \multicolumn{4}{|l|}{ Amantadine hydrochloride } \\
\hline Indication & For PD & For depression & For PD \\
\hline Dose, mg/day & 150 & 200 & 300 \\
\hline \multicolumn{4}{|l|}{ Medication period } \\
\hline Until onset & >1 year & >1 year & A few days \\
\hline From onset & 2 months & 2 months & 3 weeks \\
\hline Outcome of toxicity & Full recovery & Full recovery & Poor recovery \\
\hline \multicolumn{4}{|l|}{ Features of toxicity } \\
\hline Disturbed consciousness & + & + & + \\
\hline Hallucination & + & + & - \\
\hline Grasp reflexes & + & + & + \\
\hline Myoclonus & + & + & + \\
\hline Gait disturbance & + & + & + \\
\hline Extrapyramidal sign & $++^{1}$ & ND & $+{ }^{1}$ \\
\hline Pyramidal sign & - & - & $+^{2}$ \\
\hline Cerebellar sign & - & - & - \\
\hline \multicolumn{4}{|c|}{$\begin{array}{l}\text { CGN = Chronic glomerulonephritis; } \mathrm{DN}=\text { diabetic nephropathy; } \mathrm{PD}=\text { Parkinson's } \\
\text { disease; ND = not determined. } \\
{ }^{1} \text { The degree of parkinsonism did not change after toxicity. } \\
{ }^{2} \text { Left hemiparesis due to lacunar infarction. }\end{array}$} \\
\hline
\end{tabular}

On admission, she had severe dementia and moderate parkinsonism, such as bradykinesia and limb rigidity with left predominance, without involuntary movement. Grasp reflexes were recognized on both sides, but palmo-mental reflexes were not elicited. She also had mild left hemiparesis due to lacunar infarction in the right corona radiata, which was revealed by brain magnetic resonance imaging scans. There were also multiple cerebral infarctions in the right fronto-parietal and occipital lobes as well as multiple and confluent white matter changes. Electroencephalography demonstrated diffuse $\theta$ waves without triphasic waves. Her serum amantadine hydrochloride level was not detectable, suggesting that it had already washed out over the previous 5 months. Oral administration of $450 \mathrm{mg}$ levodopa and $0.25 \mathrm{mg}$ pramipexole per day improved her parkinsonism, but her daily living skills did not improve.

\section{Discussion}

The clinical symptoms and signs of amantadine toxicity in patients 1 and 2 were reversible after cessation of amantadine administration, whereas those in patient 3 partially improved, but ultimately persisted. The duration between the initiation and cessation of oral amantadine was 2 months in patients 1 and 2, and 3 weeks in patient 3 . Renal dysfunction was relatively mild in patient 2 , whereas patients 1 and 3 had chronic renal failure and regularly underwent hemodialysis. The doses of amantadine were 150, 200, and $300 \mathrm{mg} /$ day in patients 1,2 , and 3 , respectively. Cerebral ischemic changes were apparent in patient 3 , but not in patient 1 . The abovementioned differences among the three patients suggest that the irreversibility of toxicity in patient 3 was due to both high dose exposure combined with severe renal dysfunction and coexisting cerebral ischemic damage.
Somnolence, inability to stand and walk, myoclonic jerks in limbs, and frontal lobe signs, such as grasp reflexes, were common neurological features in all 3 patients. With regard to disturbed consciousness, somnolence was common in all 3 patients. Inability to stand and walk was caused by factors other than motor paresis, cerebellar ataxia, or parkinsonism. Myoclonic jerks were present in all patients, and were exaggerated during motion. A previous study indicated that myoclonic jerks in a patient with amantadine toxicity originated from the cerebral cortex [1]. The above-mentioned features of amantadine toxicity, such as somnolence and myoclonus, resemble those of Creutzfeldt-Jakob disease. The same clinical features have been also reported as drug-induced Creutzfeldt-Jakob-like syndrome in patients with toxicity from lithium, lithium with levodopa, antidepressants, and bismuth [2-6]. It is important to differentiate 
the consciousness disturbance in drug toxicity from the dementia in CreutzfeldtJakob disease, but this differentiation is not very easy in patients with severe dysfunction, such as patient 3 .

Amantadine hydrochloride is only available orally and is well absorbed by the intestine. It is widely distributed and stored in many organs such as the lungs and kidneys; there is a small component in the blood circulation $[7,8]$. Because approximately $90 \%$ of the drug is excreted unchanged in the urine, it accumulates in various organs in patients with renal dysfunction $[7,8]$. The half-life of drug elimination is $11.8 \pm 2.1 \mathrm{~h}$ (range: $9.7-14.5 \mathrm{~h}$ ) in subjects with normal renal function, and 8.3 days (range: $7.0-10.3$ days) in patients on hemodialysis [9]. Furthermore, the half-life in patients whose creatinine clearance is reduced to 50,30 , and $10 \mathrm{ml} / \mathrm{min}$, has been reported to be $23 \mathrm{~h}, 40 \mathrm{~h}$, and 7.4 days, respectively [9]. Because the serum creatinine level does not become elevated before creatinine clearance is reduced to $<50 \mathrm{ml} / \mathrm{min}$, amantadine excretion might be extended even in patients with a normal serum creatinine level, such as patient 2 in the present report. It is remarkable that only $2-5 \%$ of the drug in the body is removed with a single hemodialysis treatment [9].

There are no substantive experimental data regarding the relationship between plasma amantadine levels and the occurrence of amantadine toxic effects. Previ- ous clinical reports suggested that plasma amantadine concentrations $>1,000 \mathrm{ng} / \mathrm{ml}$ in humans were associated with toxic manifestations such as hallucinations, depression, confusion, dizziness, disinhibition, seizures, and cortical myoclonus [8]. The plasma amantadine hydrochloride level in patient 1 was very high at $4,900 \mathrm{ng} / \mathrm{ml}$. Striking features such as an inability to walk and frontal lobe signs, which were recognized in our patients, however, have not been described before. Amantadine toxicity from large doses might be fatal because of its cardiovascular effects, e.g., 2 deaths have occurred in patients attempting suicide with doses of 2 g/day [10-12].

In conclusion, practitioners should not use amantadine hydrochloride to medicate patients with renal failure, and extreme caution should be used when medicating older patients with possible occult renal dysfunction. The neurological features of amantadine toxicity vary, but somnolence, myoclonic jerks, and an inability to walk are considered early and important features.

\section{References}

1 Matsunaga K, Uozumi T, Qingrui L, Hashimoto T, Tsuji S: Amantadine-induced cortical myoclonus. Neurology 2001;56:279280.

2 Smith SJM, Kocen RS: A Creutzfeldt-Jakob like syndrome due to lithium toxicity. J Neurol Neurosurg Psychiatry 1988;51:120-123.
3 Broussolle E, Setiey A, Moene Y, Trielett M, Chazot G: Reversible Creutzfeldt-Jakob like syndrome induced by lithium plus levodopa treatment. J Neurol Neurosurg Psychiatry 1989;52:686-687.

4 Finelli PF: Drug-induced Creutzfeldt-Jakob like syndrome. J Psychiatr Neurosci 1992;17: 103-195.

5 Koponen H, Honkonen S, Partanen J, Riekkinen PJ: Epileptic attack, delirium, and a Creutzfeldt-Jakob-like syndrome during mianserin treatment. Neuropsychobiology 1990;23:164-168.

6 Von Bose MJ, Zaudig M: Encephalopathy resembling Creutzfeldt-Jakob disease following oral, prescribed doses of bismuth nitrate. Br J Psychiatry 1991;158:278-280.

7 Bleidner WE, Harmon JB, Hewes WE, Lynes TE, Herman EC: Absorption, distribution and excretion of amantadine hydrochloride. J Pharmacol Exp Ther 1965;150:484-490.

8 Aoki FY, Sitar DS: Clinical pharmacokinetics of amantadine hydrochloride. Clin Pharmacokinet 1988;14:35-51.

9 Horadam VW, Sharp JG, Smilack JD, McAnalley BH, Garriott JC, Stephens MK, Prati RC, Brater DC: Pharmacokinetics of amantadine hydrochloride in subjects with normal and impaired renal function. Ann Intern Med 1981;94:454-458.

10 Sartori M, Pratt CM, Young JB: Torsade de pointe: malignant cardiac arrhythmia induced by amantadine poisoning. Am J Med 1984;77:388-391.

11 Cook PE, Dermer SW, McGurk T: Fatal overdose with amantadine. Can J Psychiatry 1986;31:757-758.

12 Simpson DM, Ramos F, Ramirez LF: Death of a psychiatric patient from amantadine poisoning. Am J Psychiatry 1988;145:267-268. 